\title{
Imedos Combines High-resolution Digital Imaging with the Innovative Field of Retinal Vessel Analysis
}

a report by

Imedos

\section{DOI: 10.17925/EOR.2007.00.00.1d}

German manufacturer Imedos, the pioneer of Retinal Vessel Analysis, is built on more than 20 years of research in this area. The company created new systems for the examination of micro-vascular vessels. This offers doctors new possibilities in the field of vascular eye diseases and in the assessment of micro-vascular risk factors and indicators of systemic vascular diseases, e.g. cardio and cerebral vascular diseases.

\section{History}

Imedos is a start-up company, founded in Weimar, Germany, in 1996. One of the founders, Dr Vilser, was the head of the interdisciplinary working group for microcirculation at the Ophthalmological Department of the Friedrich Schiller University, in Jena, for a long time.

The first device of Imedos, the Retinal Vessel Analyser (RVA), was able to establish itself in the field of scientific ophthalmology. The technological break-through came with the development of the Static Vessel Analyser (SVA) and the Dynamic Vessel Analyser (DVA) in 2002/2003.

These systems were developed for clinical routine use and have paved the way for the widespread use of vessel analysis techniques. Close relationships with the Institute of Biomedical Engineering and Informatics of the Technical University of Ilmenau, university ophthalmology clinics and the Federal Government and state sponsored preparatory research programme have allowed them to make enormous progress with high innovative potential for medical science.

\section{Objectives and Visions}

Imedos' business goal is to secure and strengthen its worldwide technology and market leadership position in the field of Vessel Analysis. Imedos' vision is to make the eye an interdisciplinary window for vascular medicine.

\section{Products}

Targeted research and development activities have made Imedos an innovative manufacturer of Retinal Vessel Analysis systems. Imedos offers a future-oriented and attractive product portfolio.

The Fundus imaging system allows capturing, documenting and evaluating digital photographs of the ocular fundus. The fundus imaging line provides all the features of high-resolution fundus imaging (e.g. fluorescence angiography). This imaging device can be upgraded for static and dynamic vessel analysis.

The product line of static vessel analysis consists of the software Vesselmap2, whose basis is a single standardised fundus picture, and the SVA.

Our Vesselmap software enables us to determine the arterio-venous ratio
Figure 1: Vessel Map

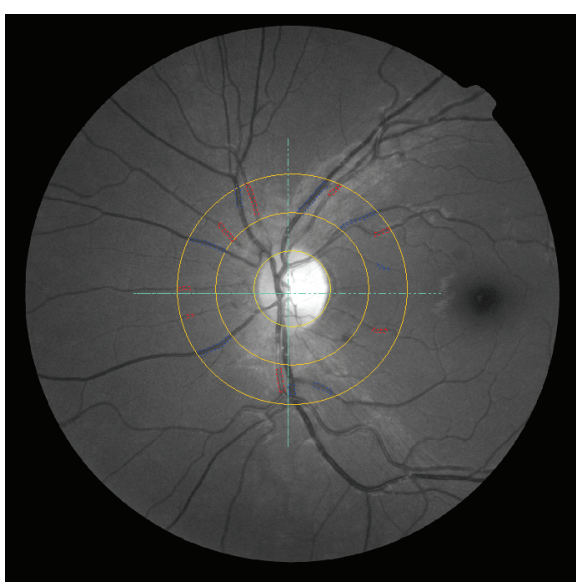

and the central arterial and venous vessel diameter equivalent in line with the various epidemiological studies, e.g. the Atherosclerosis Risk in Communities (ARIC) study. The results of these studies show that vascular changes of retinal vessels are reflected in systemic vessel changes, respectively vascular diseases. An examination report allows the medic to determine the current state of these vessels (normal, narrow or wide) and the vascular risk of the microcirculation of the retinal, as well as of the systemic circulation.

Vesselmap2 can be used together with any Fundus Imaging Systems. The combination with the Fundus Imaging supplies the SVA.

The DVA is a dynamic vessel analysis system. Dynamic vessel analysis is the key technology for the early detection of vascular dysfunctions, which again play an important role in the field of retinal and systemic vascular diseases. The DVA stimulates the microcirculation by the use of flickering light. The responses of the diameter of the arteries and veins are continuously recorded in relation to time by fundus picture sequences. The response of healthy vessels is a diameter dilation, which is estimated by the DVA. This dilation is an important functional parameter of retinal auto-regulation and allows conclusions of systemic vascular vessel functions.

\section{The Examination by the Dynamic Vessel Analyser}

The patient is positioned in front of the mydriatic fundus camera and the diameter of selected vessel segments will be measured automatically. During the measurement, flicker-light provocation of the retina is performed. The flicker-light-induced diameter changes are recorded over time for all locations along the segments. An easy-to-read graph is generated (FLIP Graph - see Figure 2), which describes the capability of the vessel to dilate. This vessel analysis is highly standardised, easy to handle and applicable in clinical routine. 
Figurer 2: Flicker Graph

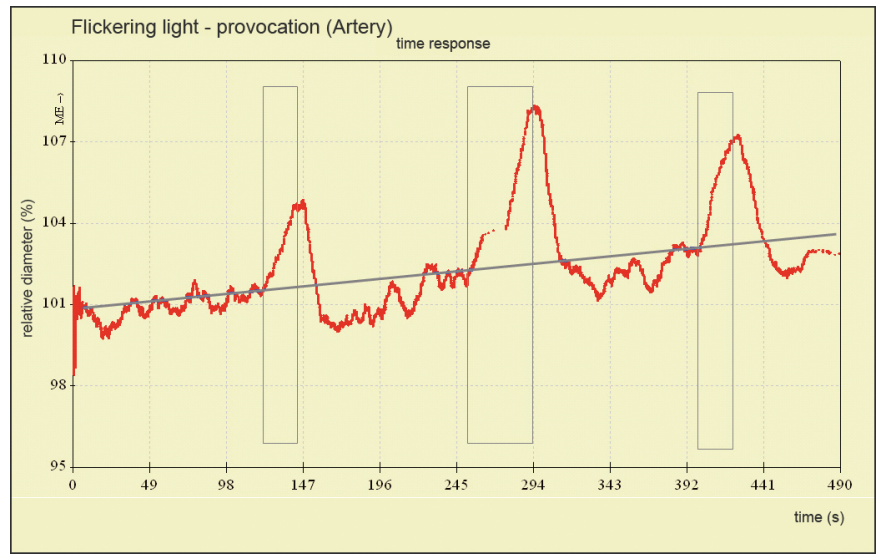

The results of the examination can be used by doctors for evaluation of vascular diseases. The DVA includes all the functions of the SVA line of
Imedos and provides all the features of high-resolution fundus imaging. Another product of dynamic vessel analysis is the RVA Research. The RVA has been developed for the needs of research institutions and practices, which do basic research or participate in clinical trials. RVA can be combined with different techniques to stimulate and examine various auto-regulation functions. As a system with unique performance parameters, it allows simple adaptation to almost any kind of clinical hypothesis in the area of vascular research. Its special software is designed to allow connections to other data sources as well as easy changes to generate different graphical data representation. The wide application fields of RVA are examinations of physiological and path physiological mechanisms or pharmacological and other therapeutic effects.

Imedos is constantly working on further valuable tools. Its modular approach to the system design will enable future upgrading. 